\title{
The Effect of Empathy on the Attentional Processing of Painful and Emotional Stimuli
}

\author{
Taiyong Bi (1D \\ Qinhong Xie ${ }^{1,2}$ \\ Jianhui Gao' \\ Tao Zhang' \\ Hui Kou'
}

'Center for Mental Health Research in School of Management, Zunyi Medical University, Zunyi, People's Republic of China; ${ }^{2}$ School of Criminal Justice, China University of Political Science and Law, Beijing, People's Republic of China
Correspondence: Taiyong Bi; Hui Kou Email bitaiyong@126.com; kouhuizmu@163.com
Background: Empathy is shown to affect the attentional processing of painful stimuli and emotional stimuli. However, whether the attentional effects on emotional stimuli depend on emotional valence and the nature of the relationship between the attentional effects on different stimuli are still unknown.

Methods: In the present study, 25 high-empathy (HE) participants and 25 low-empathy (LE) participants were recruited to perform dot-probe tasks on painful stimuli and emotional stimuli.

Results: The results showed that HE individuals had weak attentional disengagement to painful pictures. More importantly, regarding emotional pictures, HE individuals showed attentional avoidance to negative emotion pictures, while LE individuals showed attentional bias to positive emotion pictures. Correlation analysis showed that the attentional bias score and attentional disengagement score were only associated with each other within the same category of stimuli (painful, positive or negative stimuli).

Conclusion: These results revealed that HE individuals mainly showed attentional avoidance to negative stimuli, while LE individuals mainly showed attentional bias to positive stimuli.

Keywords: empathy, attentional bias, attentional disengagement, painful stimuli, emotional stimuli

\section{Introduction}

According to Rogers, ${ }^{1}$ empathy is defined as a kind of sensitive ability and willingness to understand others' thoughts, feelings and struggles from another's point of view. People seem to have "shared circuits" for self and others' pain, and this shared neural representation is associated with empathy. Previous neuroimaging studies found that when observers perceived that others were suffering from pain, the activated neural network was similar to that activated when they received painful stimulation themselves, which included the medial/anterior cingulate cortex (ACC) and anterior insula. ${ }^{2-7}$ Individual empathy is usually measured by the scale of Interpersonal Reactivity Index (IRI). The IRI score was found to be associated with the empathy for pain revealed by the vicarious pain subscale of the Empathy for Pain Scale (EPS). ${ }^{8}$ A neuroimaging study also showed that the IRI score was correlated with the neural activity in the bilateral anterior insula during state empathy for pain. ${ }^{9}$ Therefore, the scale of IRI is a suitable measurement to reveal the trait empathy and the empathy for pain.

According to the social neuroscience perspective on empathy, ${ }^{10}$ individuals' understanding of feelings and emotions in others shares the neural network that 
produces such states in themselves, which constitutes one important aspect of empathy. However, empathy, as well as its related brain activities, may be a characteristic that varies among individuals. Goubert et al ${ }^{11}$ proposed that the bottom-up processes (eg, observing person's pain expressions and contextual pain cues) and the top-down processes (eg, the observer's shared knowledge and other dispositions) influence the sense of knowing the experience of another person in pain and in turn influence the affective and behavioural responses of empathy. For example, health care professionals who are overexposed to pain stimuli may develop perceptual adaption and may thus show reduced sensitivity to pain stimuli. ${ }^{12}$ Studies revealed that these health professionals tended to underestimate patients' pain, and this tendency was more pronounced as the patients' pain severity increased. ${ }^{13-15}$ Correspondingly, neuroimaging evidence suggested that medial and superior prefrontal cortices and the temporoparietal junction involved in emotion regulation and theory of mind were activated in physicians viewing visually painful stimuli, while pain-related networks such as the anterior insula and ACC were only activated in the control group. ${ }^{16}$ The empathic trait can also modulate individuals' response for painful stimuli. Previous studies demonstrated that participant's empathy level was positively associated with their estimates for others' pain $^{17}$ and the activation in anterior insula, left inferior frontal gyrus and ACC when observers watched others' pain. ${ }^{7,18}$ Therefore, individuals with different levels of empathy may develop different processes on pain-related stimuli.

In some studies, empathy is shown to modulate attentional processing in response to painful stimuli. For example, high-empathy (HE) participants showed a longer overall gaze duration on painful faces than on neutral faces. ${ }^{19}$ Children with HE performed better on searching painful expressions among neutral expressions, indicating that the effect of empathy on the attentional processing of painful stimuli may be well developed early in life. ${ }^{20}$ However, there is other evidence showing small or moderate attentional effects on painful stimuli. A previous event-related potential (ERP) study found little difference of P200 and P300 amplitudes between painful and nonpainful stimuli. ${ }^{21}$ More importantly, a recent meta-analysis indicated a weak attentional bias towards painful pictures with the dot-probe task. ${ }^{22}$ Nevertheless, even if the attentional effect on painful stimuli is observed, the mechanism underlying the attentional processing of painful stimuli is yet to be elucidated. One possible mechanism is that individuals with HE may process emotional cues better than individuals with low empathy (LE). Evidently, painful pictures may induce more negative emotional experiences and thus bias the attention of HE individuals.

Negative emotional stimuli may capture attention even at the early stage of visual processing, suggesting a negativity bias. ${ }^{23-28}$ Some studies, in contrary, found attentional bias to positive emotional stimuli. ${ }^{28-30}$ These attentional biases could be modulated by top-down motivational significance (eg, affective mood). ${ }^{28,31-33}$ According to the social neuroscience perspective on empathy, ${ }^{10}$ empathy may have an impact on the processing of emotional stimuli. It was found that HE participants could quickly detect targets' emotions. ${ }^{34}$ Through a paradigm of the emotional Stroop effect, Hofelich and Preston $^{35}$ showed that empathy could increase attention to emotional information. Consistently, eye-movement studies found increased fixation duration on emotional faces and the most affective facial region (eyes) for $\mathrm{HE}$ individuals. $^{36-38}$ At the neural activity level, electrophysiological evidence also suggested that the level of empathy affected the wave amplitudes from early (N200) to late (last positive potential) components elicited by facial expressions. $^{34,39}$ Although these results may indicate a relationship between empathy and attentional bias to emotional stimuli such as facial expressions, whether attentional bias or attentional disengagement was different between positive and negative emotional stimuli was not examined.

The dot-probe paradigm is considered to be effective in revealing the attentional processing of visual stimuli. ${ }^{40,41}$ Quantitative indicators such as the attentional bias score $(\mathrm{ABS})^{41}$ and the attentional disengagement score (ADS) ${ }^{42}$ could be calculated from the results of this paradigm, which are accurate measures of attentional processing. Specifically, ABS is defined as the difference of performance between the invalid cue condition and the valid cue condition. If a cue appears and biases the attention simultaneously, this score might be larger than zero. ADS measures how difficult one may switch the attention away from a stimulus. It is defined as the difference between the invalid cue condition and the neutral cue condition. The larger the score, the harder one can disengage the attention from the invalid cue. These indices were adopted because they could directly reflect the attentional bias and attentional disengagement processes.

In the present study, we examined the attentional processing of both painful stimuli and emotional stimuli 
among HE and LE individuals through a modified dotprobe paradigm. Attentional bias and disengagement with the stimuli were calculated directly. First, we may expect to observe attentional bias or disengagement to painful stimuli and emotional stimuli. Next, we hypothesized that $\mathrm{HE}$ individuals may show stronger attentional bias to painful and emotional stimuli than LE individuals. In addition, HE individuals may be more difficult in disengaging their attention from the painful and emotional stimuli. More specifically, as painful stimuli may also induce negative experience, the hypotheses concerning the effects of painful stimuli and negative emotional stimuli were identical. Finally, although there were studies suggesting that empathy could modulate the attentional processing on painful stimuli and emotional stimuli, direct evidence on the relationship between the two kinds of attentional processing is not examined. To reveal the relationship between the painful empathy and the emotional empathy directly, we also aimed to examine the correlation between the attentional processing of painful stimuli and emotional stimuli. We hypothesized that there might be a high correlation between painful stimuli and negative emotional stimuli.

The present study may advance our understanding about the effect of empathy on the attentional processing of emotional and painful stimuli, and may provide critical evidence for developing interventions on the attentional deficits caused by empathic problems. First, theoretically, the relationship between the pain empathy and the emotional empathy is largely unknown. Our study could help elucidate the relationship between the two kinds of empathy. Second, as we mentioned above, the empathy of health professionals may be reduced by overexposure to painful stimuli, and thus they may underestimate the pain of patients. The findings of the present study may characterise the attention of LE individuals on painful and emotional stimuli, and provide evidence for further interventions on LE individuals. Similarly, other groups with LE such as conduct disordered or anti-social disordered may be also benefit from the present study.

\section{Materials and Methods}

\section{Participants}

One hundred forty-seven undergraduates completed the Chinese version of the Interpersonal Reactivity Index (IRI-C). In order to find a significant attentional bias effect (by comparing an attentional indicator with zero), we performed a power analysis to determine the sample size through $\mathrm{G}^{*}$ Power 3.1. At a level of medium effect size $(d=0.6)$ with a statistical power of 0.8 , the result showed that a sample of 24 subjects was required. Therefore, twenty-five individuals whose IRI-C scores were within the highest $25 \%$ (HE group) and twenty-five whose IRI-C scores were within the lowest $25 \%$ (LE group) voluntarily participated in the behavioural experiments. All of the participants were right-handed and had normal or corrected-to-normal vision and normal colour vision, and none of them had a history of neurological or psychiatric illness.

The ages of the participants ranged from 18 to 22 years. No age difference was found between the $\mathrm{HE}$ group $(M=20.00, S D=1.01)$ and LE group $(M=19.96$, $S D=0.93)(t(48)=0.146, p=0.884)$. No sex difference was noted between the HE group (15 females) and LE group (13 females) $\left(\chi^{2}=0.569, p=0.776\right)$. IRI scores were significantly different between the $\operatorname{HE}(M=60.92, S D=3.63)$ and LE $(M=35.92, \quad S D=4.07)$ groups $(t(48)=22.92$, $p<0.001)$.

\section{Ethical Approval}

All procedures performed in this study involving human participants were in accordance with the ethical standards of the Ethical Committee of Human Research at Zunyi Medical University and with the 1964 Helsinki declaration and its later amendments or comparable ethical standards. Ethical consent and informed consent was obtained from all participants before participation.

\section{Materials \\ Scale}

The IRI-C was used to measure the empathy of participants. ${ }^{43}$ It contains 22 items and encompasses four subscales, including perspective taking (eg, "I try to look at everybody's side of a disagreement before I make a decision"), personal distress (eg, “Other people's misfortunes do not usually disturb me a great deal'), fantasy (eg, "When I watch a good movie, I can very easily put myself in the place of a leading character"), and empathy concern (eg, "When I see someone being taken advantage of, I feel kind of protective towards them"). All of the items were rated on a 5-point Likert scale from 0 ("not at all like me") to 4 ("very much like me"). The Cronbach's $\alpha$ coefficient of the IRI-C was 0.75 , and its test-retest reliability was 0.74 in a Chinese sample. ${ }^{43}$ In the present 
study, the Cronbach's $\alpha$ coefficient was 0.84 . In the present study, IRI-C scores were collected from a sample of undergraduates. The combined mean score from the HE group and the LE group was 48.35 ( $\mathrm{SD}=9.15)$. We compared our scores with other studies using the IRI scale in Chinese groups. In healthy adults, the score ranged from 48.40 to $51.50 .^{43-45}$ In undergraduates, the scores were 52.97, 47.50, 48.56 and 51.20 for normal students, medical school students, nursing students and military school students, respectively. ${ }^{46-49}$ The score in the present study was comparable with those of previous studies. More importantly, in our study, the HE group showed an average score of 60.92 on the IRI scale, while the LE group showed an average score of 35.92 , which was significantly different from the average scores of the present study and previous studies. These results demonstrated that the selection of HE and LE individuals was appropriate.

\section{Painful Pictures}

Digital colour pictures depicting a model's hand, forearm, or foot in painful or nonpainful situations were adopted from a previous study and presented as stimuli. ${ }^{50}$ Eighteen painful pictures and 42 nonpainful pictures were selected, forming 18 painful-nonpainful picture pairs and 12 nonpainful-nonpainful pairs. All pictures had a uniform size (354*266 pixels) and were matched for brightness and contrast. The pain intensity of each picture was evaluated by a group of 70 undergraduates on a 9 -point scale (" $1=$ no sensation" to "9=unbearable pain"). The results showed significantly different intensities between the painful $(M=6.534, \quad S D=1.023) \quad$ and nonpainful $(M=2.162$, $S D=0.470)$ pictures $(t(69)=22.80, p<0.001)$. The results regarding emotional valence ( $1=$ very unhappy, $9=$ very happy) revealed significant difference between the painful $(M=2.315, \quad S D=0.715) \quad$ and nonpainful $\quad(M=4.498$, $S D=1.480)$ pictures $(t(69)=5.95, p<0.001)$.

\section{Emotional Pictures}

Digital colour pictures depicting emotional situations were adopted from the Chinese Affective Picture System (CAPS) ${ }^{51}$ Twenty-four positive, 24 negative and 72 neutral emotional pictures were selected. All pictures had a uniform size $\left(433^{*} 315\right.$ pixels $)$ and were matched for brightness and contrast. The valence and arousal of each picture were evaluated by a group of 46 undergraduates on a 9-point scale. Kruskal-Wallis test revealed that the emotional valence was significantly different among positive $(M=7.36, S D=0.28)$, negative $(M=1.76, S D=0.27)$ and neutral $(M=4.45, S D=0.56)$ pictures $(p<0.001)$. Post hoc tests showed significant difference between each two kinds of pictures (all $p<0.001$, Bonferroni corrected). KruskalWallis test revealed that the arousal was significantly different among positive $(M=6.40, S D=0.41)$, negative $(M=6.46, S D=0.29)$ and neutral $(M=4.15, S D=0.59)$ pictures $(p<0.001)$. Post hoc tests showed significant lower arousal of neutral pictures than positive and negative pictures (all $p<0.001$, Bonferroni corrected), while no significant difference was found between the arousal of positive and negative pictures $(p>0.05)$.

\section{Apparatus}

The visual stimuli were presented on a SAMSUNG 19-in LCD screen, with a spatial resolution of $1280 \times 800$ and a refresh rate of $60 \mathrm{~Hz}^{52}$ The subjects viewed the stimuli from a distance of $60 \mathrm{~cm}$. The presentation of stimuli was controlled by E-Prime 2.0 software (https://www.pstnet.

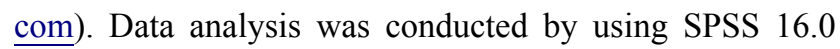
(https://www.ibm.com/analytics/SPSS-statistics-software).

\section{Procedure}

A modified version of the dot-probe paradigm was adopted. The procedure is demonstrated in Figure 1.

\section{Experiment I: Attentional Bias to Painful Pictures}

At the beginning of each trial, a white fixation cross with a black background was presented in the centre of the screen for a random period of 500 1000 ms. Afterwards, a pair of pictures was presented for $500 \mathrm{~ms}$, with one picture on each side of the visual field. A probe was presented immediately after the disappearance of the pictures. The probe consisted of three horizontally aligned white dots or three vertically aligned white dots, whose centre was coincident with one of the pictures. Participants were asked to press one key ( $\mathrm{F}$ or $\mathrm{J}$ ) if the dots were horizontally aligned and another key (J or F) if the dots were vertically aligned as quickly and accurately as possible. The painful cue was valid if the locations of the painful picture and the probe were the same. Otherwise, the cue was invalid. Each probe appeared until a response was made within $5 \mathrm{~s}$, followed by a blank screen for 500 ms. Each participant completed 2 experimental blocks. Each block consisted of 96 trials, including 24 trials of nonpainful-nonpainful pairs, 24 trials of painfulnonpainful pairs with hand injury, 24 trials of painfulnonpainful pairs with forearm injury, and 24 trials of painful-nonpainful pairs with foot injury. In total, each 


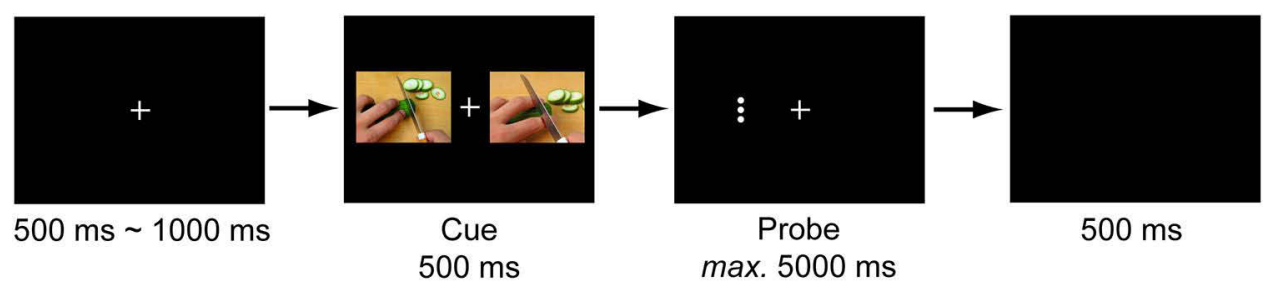

Figure I An example of the experimental procedure of dot-probe paradigm. A cue consisted of two pictures. In cueing trials, one of the pictures was a painful/emotional picture, while the other one was a nonpainful/neutral picture. In non-cueing trials, both pictures were nonpainful/neutral pictures. A probe was presented immediately after the disappearance of the cue, and persisted until the participant made a response within 5000 ms. Participants were asked to judge the orientation of the probes.

participant completed 48 trials of nonpainful-nonpainful pairs and 144 trials of painful-nonpainful pairs. The types of picture pairs, positions of painful pictures, and positions of probes were all counterbalanced in each block. The type of key press (eg, F for a horizontally aligned probe or $\mathrm{J}$ for a horizontally aligned probe) was randomly assigned to each participant.

\section{Experiment 2: Attentional Bias to Emotional Pictures} The procedure of Experiment 2 is similar to that of Experiment 1, except that the stimuli were changed to emotional pictures. The emotional cue was valid if the locations of the emotional picture and the probe were the same. Otherwise, the cue was invalid. Each participant completed 3 experimental blocks. Each block consisted of 48 trials, including 16 positive-neutral pairs, 16 negative-neutral pairs and 16 neutral-neutral pairs. In total, each participant completed 48 trials of positive-neutral pairs, 48 trials of negative-neutral pairs, and 48 trials of neutral-neutral pairs. Positions of emotional pictures and probes were also counterbalanced in each block.

\section{Statistical Analysis}

The raw RT data were preprocessed by excluding outliers beyond or below the mean with three standard deviations. To directly reveal the attentional bias to and the attentional disengagement to painful or emotional pictures, we calculated the $\mathrm{ABS}$ and $\mathrm{ADS}$ for each experiment.

The ABS was calculated according to previous studies. ${ }^{41,53}$ This index was calculated based on the reaction times (RTs) to probes at consistent or inconsistent positions with painful or emotional pictures. In Experiment 1, ABS $=[($ PlDr-PrDr $)+($ PrDl-PlDl $)] / 2$ ( $\mathrm{P}=$ Painful pictures, $\mathrm{D}=$ Dot-probe, $\mathrm{l}=$ left and $\mathrm{r}=$ right $)$. A positive $\mathrm{ABS}$ indicates that attention is biased to the painful picture (ie, faster responses to probes following painful pictures than to probes following nonpainful pictures). In contrast, a negative ABS indicates attentional avoidance of the painful picture. In Experiment 2, for positive pictures, $\mathrm{ABS}=[(\mathrm{PlDr}-\mathrm{PrDr})+(\mathrm{PrDl}-\mathrm{PlDl})] / 2$ $(\mathrm{P}=$ Positive emotional pictures, $\mathrm{D}=$ Dot-probe, $\mathrm{l}=\mathrm{left}$ and $\mathrm{r}=$ right $)$; for negative pictures, $\mathrm{ABS}=[(\mathrm{NlDr}-\mathrm{NrDr})$ $+(\mathrm{NrDl}-\mathrm{NlDl})] / 2(\mathrm{~N}=$ Negative emotional pictures, $\mathrm{D}=$ Dotprobe, $\mathrm{l}=$ left and $\mathrm{r}=$ right).

The ADS was calculated according to methods used in previous studies. ${ }^{42,54}$ This index was calculated based on the RTs to probes at inconsistent positions with painful or emotional pictures and the RTs to probes following nonpainful or neutral pairs of pictures. In Experiment 1, ADS $=(\mathrm{PlDr}+\mathrm{PrDl}) / 2-(\mathrm{NDl}+\mathrm{NDr}) / 2 \quad(\mathrm{P}=$ Painful pictures, $\mathrm{D}=$ Dot-probe, $\mathrm{N}=$ Nonpainful pictures pair, $\mathrm{l}=\mathrm{left}$ and $r=$ right). A positive ADS indicates that one has difficulty disengaging their attention from painful pictures. In Experiment 2, for positive pictures, $\mathrm{ADS}=(\mathrm{PlDr}+\mathrm{PrDl}) /$ $2-(\mathrm{NeuDl}+\mathrm{NeuDr}) / 2 \quad(\mathrm{P}=$ Positive emotional pictures, $\mathrm{D}=$ Dot-probe, Neu=Neutral pictures pair, $\mathrm{l}=\mathrm{left}$ and $\mathrm{r}=$ right); for negative pictures, $\mathrm{ADS}=(\mathrm{NeglDr}+\mathrm{NegrDl}) /$ $2-(\mathrm{NeuDl}+\mathrm{NeuDr}) / 2$ (Neg=Negative emotional pictures, $\mathrm{D}=$ Dot-probe, $\mathrm{Neu}=$ Neutral pictures pair, $\mathrm{l}=\mathrm{left}$ and $\mathrm{r}=$ right).

In Experiment 1, independent $t$-tests were conducted on the ABS and ADS separately to examine the group difference. In addition, comparisons between the ABS/ADS and zero were performed in each group to examine whether there was significant attentional bias or attentional disengagement to the painful pictures. In Experiment 2, two 2 (valence: positive/negative) * 2 (group: HE/LE) mixed ANOVAs were first conducted on ABS and ADS. In addition, direct comparisons between the ABS/ADS and zero were also performed in each group and each condition to examine whether there was attentional bias or attentional disengagement in the emotional pictures. Finally, Pearson correlational analyses were performed on the scores of ABS and ADS to 
examine whether there were relationships between ABSs and ADSs and between scores from different tasks.

\section{Results}

\section{Accuracy Results}

In the two experiments, accuracies were quite high in all of the conditions in each group (all $M>95 \%, S D<6 \%$, see Tables S1 and $\underline{\text { S2}}$ ), indicating that participants paid considerable attention to the tasks and that they did not sacrifice accuracy for faster responses. More importantly, we performed a 3 (condition: valid/invalid/neutral) $\times 2$ (group: HE/LE) mixed ANOVA for Experiment 1 and a 5 (condition: positive valid/positive invalid/negative valid/negative invalid/neutral) $\times 2$ (group: HE/LE) mixed ANOVA for Experiment 2 on the accuracy data. Results showed no significant interactions and main effects in either Experiment 1 or Experiment 2 (all $F<1.4, p>0.2$ ).

\section{RT Results}

The RTs and statistical results from Experiment 1 are displayed in Tables S3 and S4. The RTs and statistical results from Experiment 2 are displayed in Tables S5-S7.

\section{Results from Experiment I}

We compared the ABS and the ADS between the HE and LE groups (Figure 2). The results showed nonsignificant group differences in the ABS $(t(48)=0.943, p=0.350$, Cohen's $\mathrm{d}=0.266)$ and $\operatorname{ADS}(t(48)=0.963, p=0.340$, Cohen's $d=0.272$ ).

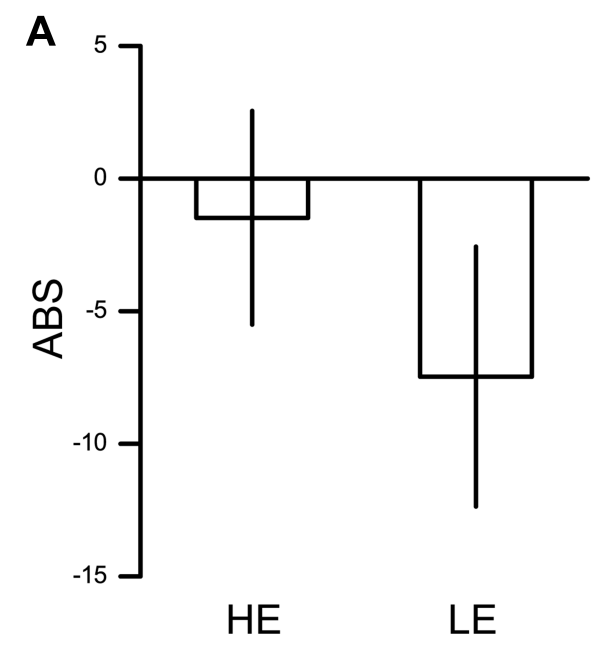

Then, we directly compared the ABS or ADS with zero in each group. In the HE group, the ABS was not significantly different from zero $(t(24)=-0.367, p=0.717$, Cohen's $d=0.073)$. ADS was significantly higher than zero in the HE group $(t(24)=2.290, p=0.031$, Cohen's $\mathrm{d}=0.458$ ), indicating difficulty in disengaging attention from painful pictures in HE individuals. In the LE group, neither the ABS nor ADS was found to be significantly different from zero (ABS: $t(24)=-1.522, p=0.141$, Cohen's $\mathrm{d}=0.304$; ADS: $t(24)=1.537, p=0.137$, Cohen's $\mathrm{d}=0.307$ ).

\section{Results from Experiment 2}

We first examined the ABSs (Figure 3A). There was no significant interaction effect between valence and group ( $F$ $\left.(1,48)=0.196, p=0.660, \eta_{p}^{2}=0.004\right)$. The main effect of valence was significant $(F(1,48)=8.480, \quad p=0.005$, $\left.\eta_{p}^{2}=0.150\right)$, indicating that ABSs were higher for positive pictures. The main effect of group was also significant $(F(1$, $\left.48)=5.133, p=0.028, \eta_{p}^{2}=0.097\right)$, indicating that ABSs were higher for the LE group. Direct comparisons between ABSs and zero were performed in each group. In the HE group, ABSs were lower than zero only for negative pictures $(t(24)$ $=-2.874, p=0.008$, Cohen's $\mathrm{d}=0.575)$, but not for positive pictures $(t(24)=0.225, p=0.824$, Cohen's $d=0.045)$. In the LE group, ABSs were higher than zero only for positive pictures $(t(24)=2.342, p=0.028$, Cohen's $d=0.468)$, but not for negative pictures $(t(24)=-0.451, p=0.656$, Cohen's $\mathrm{d}=0.090$ ). These results indicated that HE individuals had attentional avoidance to negative pictures and LE individuals had attentional bias to positive pictures.

B

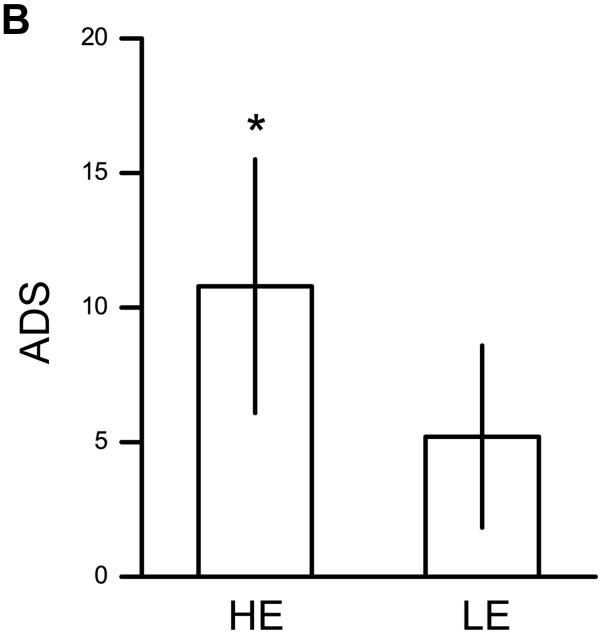

Figure 2 Results from Experiment I. (A) Averaged ABSs for painful stimuli in HE and LE groups. (B) Averaged ADSs for painful stimuli in HE and LE groups. Error bar denotes I standard error of mean. ${ }^{*} p<0.05$.

Abbreviations: ABS, attentional bias score; ADS, attentional disengagement score; HE, high empathy; LE, low empathy. 
A

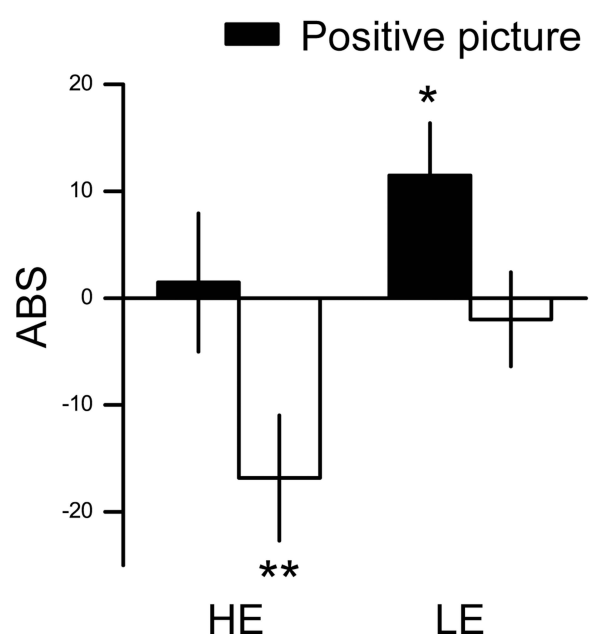

B

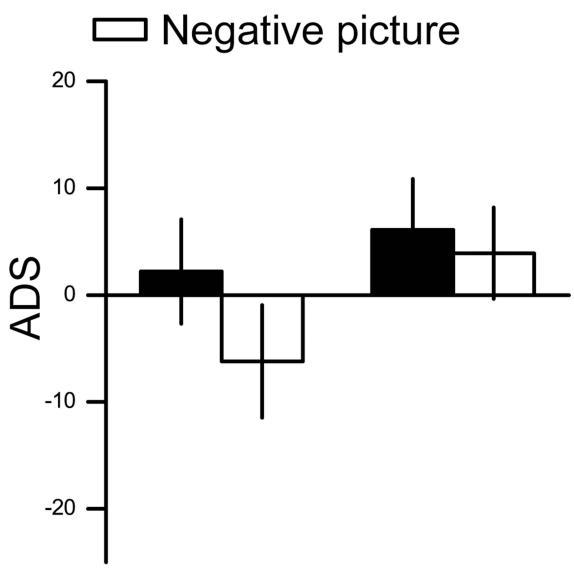

HE LE

Figure 3 Results from Experiment 2. (A) Averaged ABSs for emotional stimuli in HE and LE groups. (B) Averaged ADSs for emotional stimuli in HE and LE groups. Error bar denotes I standard error of mean. $* p<0.05$, $* * p<0.0$ I.

Abbreviations: $A B S$, attentional bias score; ADS, attentional disengagement score; HE, high empathy; LE, low empathy.

For ADSs (Figure 3B), no significant interaction effect or main effects were found (interaction: $F(1,48)$ $=1.660, p=0.204, \eta_{p}^{2}=0.033$; emotion valence: $F(1,48)$ $=0.575, p=0.452, \eta_{p}^{2}=0.012 ;$ group: $F(1,48)=1.666$, $p=0.203, \eta_{p}^{2}=0.034$ ). The ADSs for each group and each condition were also not different from zero (positive/HE: $t(24)=0.450, p=0.657$, Cohen's $\mathrm{d}=0.090$; negative/HE: $t(24)=-1.179, \quad p=0.250$, Cohen's $\mathrm{d}=0.236$; positive/LE: $t(24)=1.279, p=0.213$, Cohen's $\mathrm{d}=0.256$; negative/LE: $t(24)=0.914, p=0.370$, Cohen's $\mathrm{d}=0.183$ ).

\section{Correlational Results}

The correlational results are displayed in Table 1. In the HE group, correlation analysis only showed a significant relationship between ABSs and ADSs of negative pictures ( $r=0.603, p=0.015$, FDR corrected). In the LE group, correlation analysis showed that 1) the relationship between the ABS and ADS of painful pictures was significant ( $r=0.522, p=0.040$, FDR corrected), 2) the relationship between the ABS and ADS of positive pictures was significant ( $r=0.645, p=0.015$, FDR corrected), 3 ) and

Table I Correlational Results

\begin{tabular}{|c|c|c|c|c|c|c|c|}
\hline & & \multicolumn{2}{|c|}{ Painful Stimuli } & \multicolumn{4}{|c|}{ Emotional Stimuli } \\
\hline & & IABS & 2ADS & 3ABS_P & 4ADS_P & 5ABS_N & 6ADS_N \\
\hline HE group & $\begin{array}{l}1 \\
2 \\
3 \\
4 \\
5 \\
6\end{array}$ & $\begin{array}{c}- \\
0.125 \\
0.095 \\
-0.171 \\
-0.175 \\
-0.050\end{array}$ & $\begin{array}{c}- \\
0.117 \\
0.260 \\
-0.164 \\
-0.101\end{array}$ & $\begin{array}{c}- \\
0.210 \\
0.123 \\
-0.149\end{array}$ & $\begin{array}{c}- \\
0.166 \\
0.297\end{array}$ & $\begin{array}{c}- \\
0.603^{* *}\end{array}$ & - \\
\hline LE group & $\begin{array}{l}1 \\
2 \\
3 \\
4 \\
5 \\
6\end{array}$ & $\begin{array}{c}- \\
0.522^{* *} \\
-0.267 \\
-0.193 \\
-0.291 \\
-0.379\end{array}$ & $\begin{array}{c}- \\
0.114 \\
0.109 \\
-0.140 \\
-0.084\end{array}$ & $\begin{array}{c}- \\
0.645 * * * \\
-0.199 \\
-0.098\end{array}$ & $\begin{array}{c}- \\
-0.179 \\
0.244\end{array}$ & $\begin{array}{c}- \\
0.521^{* *}\end{array}$ & - \\
\hline
\end{tabular}

Notes: The six numbers in each group correspond to the six variables listed under painful and emotional stimuli. $* * p<0.01$, $* * * p<0.001$, uncorrected.

Abbreviations: $A B S$, attentional bias score; $A D S$, attentional disengagement score; $P$, positive picture; $N$, negative picture. 
the relationship between ABS and ADS of negative pictures was significant ( $r=0.521, p=0.040$, FDR corrected).

These results indicated that ABSs and ADSs were associated with each other only in tasks with the same stimuli, supporting that there might be different processing methods for different stimuli.

\section{Discussion}

In the present study, we examined the impact of empathy on the attentional processing of painful and emotional stimuli with a modified dot-probe paradigm. Significant attentional effects were observed in some conditions, demonstrating the validity of the task. Two hypotheses concerning the effect of empathy were proposed. First, HE individuals may show stronger attentional processing on painful and emotional stimuli than LE individuals. Second, the attentional processing of painful stimuli may have a close relationship with that of negative emotional stimuli, ie, they are significantly correlated with each other. These hypotheses were partly supported. First, we found that HE individuals but not LE individuals showed a trend of attentional disengagement to painful stimuli. Second, HE individuals showed attentional avoidance to negative emotional stimuli, while LE individuals showed attentional bias to positive emotional stimuli. These results indicate that HE and LE individuals may have different attentional processing of painful and emotional stimuli. Furthermore, correlational analysis showed that ABSs and ADSs were associated with each other only within the same category of stimuli, indicating that the attentional processing of different stimuli may not share a common mechanism.

In Experiment 1, we only observed a relatively weak result showing that $\mathrm{HE}$ individuals showed difficulty in disengaging their attention from painful pictures, which may indicate attentional maintenance to painful information. This finding was consistent with previous findings on painful expressions, which revealed that HE participants showed overall attentional maintenance to painful expressions relative to nonpainful expressions. ${ }^{19}$ However, no significant attentional bias to the painful stimuli was found in our study, which is surprising and seems contradictory to our hypothesis. Researchers have proposed that painful stimuli may attract observers' attention ${ }^{55}$ and found that brain areas including the ACC/paracingulate and the right middle frontal gyrus were activated only when the painful stimuli were explicitly attended. ${ }^{56}$ These findings indicate an important role of attention in the processing of painful stimuli. A potential reason for the discrepancy between previous findings and ours might be that attentional bias of painful stimuli may rely on the emotional arousal of pictures. That is, if the painful stimulus fails to induce emotional experience, it may also fail to attract the attention. Previous studies revealed that empathy was closely related to empathic emotion ${ }^{57}$ and that individuals experiencing high levels of empathic emotion were ready to offer help to victims when watching other people being given electric shocks. ${ }^{58}$ Emotional stimuli have been revealed to be effective in biasing visual attention automatically and enhancing brain activities in the visual cortex. ${ }^{59,60}$ Therefore, attentional bias may be induced by painful stimuli only when they are fully processed and emotional information is successfully extracted, which might not have been fully achieved in the present study as they were only presented for $500 \mathrm{~ms}$. Further studies are required to examine the attentional bias induced by painful stimuli by adopting a longer presentation duration. In addition, we should also note that a larger ADS may result from a general slowing of response to the painful stimulus, which should be ruled out in future studies.

More importantly, in Experiment 2, we found that HE participants showed attentional avoidance of negative emotional pictures while LE participants did not. It could be a protective mechanism for HE individuals, as they may experience more intense feelings and emotions than LE individuals. Individuals with $\mathrm{HE}$ were found to express stronger negative emotional reactions to negative pictures showing aggressive acts. ${ }^{61}$ Compared with highly threatening stimuli, those with a moderate level of threat may be more effective on attentional attraction than attentional avoidance. ${ }^{62,63}$ For example, a threat expressed by facial expression is moderately intense, and HE individuals were found to be better in recognizing expressions and showed response bias towards sad and fearful expressions. ${ }^{64}$ In the present study, vivid negative pictures were adopted, which may induce a more intense emotion than facial expressions. As a result, HE individuals showed attentional avoidance rather than attraction to these negative pictures.

Meanwhile, we also found that LE individuals showed attentional bias towards positive emotional pictures. It was not surprising, as such an effect was consistently found in previous studies. ${ }^{65}$ According to the empathy-altruism hypothesis, high level of empathy may induce altruistic motivation. ${ }^{58,66}$ Therefore, the LE individuals may have more self-interested rather than altruistic motivated, and 
may thus be motivated by self-interested hedonism to show attentional bias towards positive emotional pictures. From another aspect, the present measurement of empathy may mainly measure the negative empathy which refers to understanding and sharing others' negative emotions. ${ }^{67}$ If the LE individuals have high level of positive empathy, they may show attentional bias towards positive emotional pictures. Future studies may test this hypothesis with measurements of positive empathy. However, our results did not reveal attentional bias on negative emotional stimuli, which seems inconsistent with previous studies showing attentional bias to emotional stimuli through eyemovement ${ }^{68,69}$ and ERP $^{70}$ approaches. There might be two explanations for this finding. First, negative stimuli could not elicit attention among LE individuals, as they may perceive emotion weakly. For example, a previous study found that empathy could enhance attention to emotional information. ${ }^{35}$ However, it may be hard to explain why LE individuals showed attentional bias to positive emotional stimuli. Second, negative stimuli also induced attentional avoidance, which compromised the attentional bias outcome observed among LE individuals. As we found significant attentional avoidance among $\mathrm{HE}$ individuals, such a process was highly possible to affect LE individuals as well. The two processes of attentional bias and attentional avoidance induced by negative stimuli should be examined in further studies.

Finally, we did not find a strong relationship between performance regarding painful stimuli and emotional stimuli. Evidence suggested that painful stimuli seemed to modulate visual attention more weakly than emotional stimuli. An ERP study revealed that painful stimuli elicited larger P3 (380-500 ms) components than neutral stimuli only when subjects were performing a pain judgement task, not when they were performing a counting task. $^{71,72}$ Therefore, painful stimuli processing may require high attentional investment. In contrast, emotional stimuli processing was found to be mostly automatic and unconscious. $^{73,74}$ Furthermore, performance regarding positive stimuli was not associated with that for negative stimuli. Consistently, previous ERP studies found that the time course of the neural response to positive stimuli was largely different from that to negative stimuli. ${ }^{30}$ Although our study did not reveal the relationship between different tasks, further studies are required to investigate whether the processing of emotional stimuli and painful stimuli share common mechanisms that could be revealed by adapting other tasks.
We noticed that there are several limitations in the present study. First, participants in the present study were divided into $\mathrm{HE}$ and $\mathrm{LE}$ groups based on their general empathy trait rather than a specific dimension of the trait. It is possible that different kinds of empathy, such as the cognitive empathy (understanding others' emotion) and the emotional empathy (sharing others' emotion), may play different roles in the attentional processing of painful and emotional stimuli. For example, the total fixation durations on painful, happy and fearful video clips were not different between controls and individuals with impaired affective empathy but normal cognitive empathy, indicating that affective empathy may not influence the attentional maintenance on emotional stimuli. ${ }^{75}$ In contrary, participants with high level of affective empathy were found to show attentional bias to emotional words. ${ }^{35}$ Further studies may examine the roles of different kinds of empathy in different attentional processes. Second, in a few studies with dotprobe task, relatively low internal consistency and retest reliability over one week were found for the measurement. ${ }^{76-78}$ The reliability of dot-probe task could be modulated by some factors. For example, the reliability of dot-probe task was stronger when the target was presented at the bottom visual field, and was stronger in later sessions in an experiment. ${ }^{79,80}$ Therefore, the dot-probe task might be unsuitable for measuring individual difference. However, evidence showed that the group difference revealed by the dot-probe task was much more reliable. ${ }^{78}$ Nevertheless, more evidences are needed to validate our findings. Furthermore, although we did not find a strong attentional effect with the current paradigm, such an effect might be revealed in other paradigms. For example, an eyemovement study showed that $\mathrm{HE}$ individuals focused on painful faces longer than they focused on neutral faces, which indicated strong sustained attention on painful stimuli in HE individuals. ${ }^{19}$ Our results also implied a similar trend by showing that $\mathrm{HE}$ individuals had difficulty in attentional disengagement from painful stimuli, although the effect was relatively weak. Further studies are required to investigate the effect of empathy on the attentional processing of painful stimuli through other paradigms, especially those related to sustained attention. Third, we measured the empathy of participants by a subjective self-report scale, which might not reflect the objective empathy level of individuals. The present findings might be affected if we measure empathy more objectively. However, objective measurements on empathy are still yet to be developed. For examples, heart rate and skin conductance level in response to emotional 
stimuli were considered as indicators of affective empathy. ${ }^{75,81-84}$ It was found that individuals with impaired affective empathy showed reduced heart rate to painful and fearful stimuli and reduced skin conductance level to painful stimuli. ${ }^{75}$ Besides, behavioural performance of emotional understanding in response to emotional stimuli was considered as the index of cognitive empathy. ${ }^{75,85}$ For example, patients with behavioural variant frontotemporal dementia showed deficiency in inferring mental states for emotional persons, indicating a cognitive empathy deficit. ${ }^{86}$ Future studies may measure participants' empathy with both subjective and objective measurements, and examine the relationship and difference between the two kinds of measurements.

In conclusion, in the present study, we found that $\mathrm{HE}$ individuals showed attentional avoidance of negative emotional pictures and had difficulty disengaging their attention from painful pictures, while LE individuals showed attentional bias towards positive emotional pictures. These results imply that different means of processing painful and emotional pictures exist for HE and LE individuals.

\section{Funding}

This work was supported by Research Project of Center for Medical Humanities in Guizhou Universities (2019jd035, 2020jd012), the New Scientist Training Program of Zunyi Medical University ([2017]5733-014), the Scientific Research Startup Foundation of Zunyi Medical University (F-875; F-990), and the National Natural Science Foundation of China (32060191).

\section{Disclosure}

The authors declare no potential conflicts of interest with respect to the research, authorship, and/or publication of this article.

\section{References}

1. Rogers CR. A Way of Being. Boston: Houghton Mifflin; 1980.

2. Danziger N, Faillenot I, Peyron R. Can we share a pain we never felt? Neural correlates of empathy in patients with congenital insensitivity to pain. Neuron. 2009;61(2):203-212. doi:10.1016/j.neuron.2008. 11.023

3. Decety J, Michalska KJ, Akitsuki Y. Who caused the pain? An fMRI investigation of empathy and intentionality in children. Neuropsychologia. 2008;46(11):2607-2614. doi:10.1016/j. neuropsychologia.2008.05.026

4. Jackson PL, Brunet E, Meltzoff AN, Decety J. Empathy examined through the neural mechanisms involved in imagining how I feel versus how you feel pain. Neuropsychologia. 2006;44(5):752-761. doi:10.1016/j.neuropsychologia.2005.07.015
5. Lamm C, Decety J, Singer T. Meta-analytic evidence for common and distinct neural networks associated with directly experienced pain and empathy for pain. Neuroimage. 2011;54(3):2492-2502. doi:10.1016/j.neuroimage.2010.10.014

6. Lloyd D, Di Pellegrino G, Roberts N. Vicarious responses to pain in anterior cingulate cortex: is empathy a multisensory issue? Cogn Affect Behav Neurosci. 2004;4(2):270-278. doi:10.3758/CABN. 4.2.270

7. Singer T, Seymour B, O'doherty J, Kaube H, Dolan RJ, Frith CD. Empathy for pain involves the affective but not sensory components of pain. Science. 2004;303(5661):1157-1162. doi:10.1126/science. 1093535

8. Giummarra MJ, Fitzgibbon BM, Georgiou-Karistianis N, et al. Affective, sensory and empathic sharing of another's pain: the empathy for pain scale. Eur J Pain. 2015;19(6):807-816. doi:10.1002/ ejp.607

9. Li Y, Zhang T, Li W, Zhang J, Jin Z, Li L. Linking brain structure and activation in anterior insula cortex to explain the trait empathy for pain. Hum Brain Mapp. 2020;41(4):1030-1042. doi:10.1002/ hbm. 24858

10. Decety J, Jackson PL. A social-neuroscience perspective on empathy. Curr Dir Psychol Sci. 2006;15(2):54-58. doi:10.1111/j.09637214.2006.00406.x

11. Goubert L, Craig KD, Vervoort T, et al. Facing others in pain: the effects of empathy. Pain. 2005;118(3):285-288. doi:10.1016/j. pain.2005.10.025

12. Prkachin KM, Rocha EM. High levels of vicarious exposure bias pain judgments. J Pain. 2010;11(9):904-909. doi:10.1016/j.jpain.2009. 12.015

13. Kappesser J, Williams AC, Prkachin KM. Testing two accounts of pain underestimation. Pain. 2006;124(1-2):109-116. doi:10.1016/j. pain.2006.04.003

14. Gleichgerrcht E, Decety J. The relationship between different facets of empathy, pain perception and compassion fatigue among physicians. Front Behav Neurosci. 2014;8:243. doi:10.3389/ fnbeh.2014.00243

15. Seers T, Derry S, Seers K, Moore RA. Professionals underestimate patients' pain: a comprehensive review. Pain. 2018;159(5):811-818. doi:10.1097/j.pain.0000000000001165

16. Cheng Y, Lin C-P, Liu H-L, et al. Expertise modulates the perception of pain in others. Curr Biol. 2007;17(19):1708-1713. doi:10.1016/j. cub.2007.09.020

17. Green AD, Tripp DA, Sullivan MJL, Davidson M. The relationship between empathy and estimates of observed pain. Pain Med. 2009;10 (2):381-392. doi:10.1111/j.1526-4637.2009.00563.x

18. Saarela MV, Hlushchuk Y, Williams AC, Schürmann M, Kalso E, Hari R. The compassionate brain: humans detect intensity of pain from another's face. Cereb Cortex. 2007;17(1):230-237. doi:10.1093/ cercor/bhj141

19. Yan Z, Wang F, Su Y. The influence of empathy on the attention process of facial pain expression: evidence from eye tracking. J Psychol Sci. 2016;39:573-579.

20. Yan Z, Pei M, Su Y. Children's empathy and their perception and evaluation of facial pain expression: an eye tracking study. Front Psychol. 2017;8:2284. doi:10.3389/fpsyg.2017.02284

21. Miltner W, Johnson R, Braun C, Larbig W. Somatosensory event-related potentials to painful and non-painful stimuli: effects of attention. Pain. 1989;38(3):303-312. doi:10.1016/0304-3959(89) 90217-0

22. Todd J, van Ryckeghem DML, Sharpe L, Crombez G. Attentional bias to pain-related information: a meta-analysis of dot-probe studies. Health Psychol Rev. 2018;12(4):419-436. doi:10.1080/174371 99.2018.1521729

23. Eastwood JD, Smilek D, Merikle PM. Negative facial expression captures attention and disrupts performance. Percept Psychophys. 2003;65(3):352-358. doi:10.3758/BF03194566 
24. Smith NK, Cacioppo JT, Larsen JT, Chartrand TL. May I have your attention, please: electrocortical responses to positive and negative stimuli. Neuropsychologia. 2003;41(2):171-183. doi:10.1016/S00283932(02)00147-1

25. Carretié L, Mercado F, Tapia M, Hinojosa JA. Emotion, attention, and the 'negativity bias', studied through event-related potentials. Int $J$ Psychophysiol. 2001;41(1):75-85. doi:10.1016/S01678760(00)00195-1

26. Huang Y-X, Luo Y-J. Temporal course of emotional negativity bias: an ERP study. Neurosci Lett. 2006;398(1):91-96. doi:10.1016/j. neulet.2005.12.074

27. Norris CJ. The negativity bias, revisited: evidence from neuroscience measures and an individual differences approach. Soc Neurosci. 2019;16:1-15.

28. Hilgard J, Weinberg A, Hajcak Proudfit G, Bartholow BD. The negativity bias in affective picture processing depends on top-down and bottom-up motivational significance. Emotion. 2014;14 (5):940-949. doi:10.1037/a0036791

29. Franken IHA, Muris P, Nijs I, van Strien JW. Processing of pleasant information can be as fast and strong as unpleasant information: implications for the negativity bias. Neth J Psychol. 2008;64 (4):168-176. doi:10.1007/BF03076419

30. Carretié L, Hinojosa JA, Martín-Loeches M, Mercado F, Tapia M. Automatic attention to emotional stimuli: neural correlates. Hum Brain Mapp. 2004;22(4):290-299. doi:10.1002/hbm.20037

31. Wadlinger HA, Isaacowitz DM. Positive mood broadens visual attention to positive stimuli. Motiv Emot. 2006;30(1):87-99. doi:10.1007/ s11031-006-9021-1

32. Dai Q, Wei J, Shu X, Feng Z. Negativity bias for sad faces in depression: an Event-Related Potential Study. Clin Neurophysiol. 2016;127(12):3552-3560. doi:10.1016/j.clinph.2016.10.003

33. Kaspar K, Gameiro RR, König P. Feeling good, searching the bad: positive priming increases attention and memory for negative stimuli on webpages. Comput Human Behav. 2015;53:332-343. doi:10.1016/ j.chb.2015.07.020

34. Balconi M, Canavesio Y. Is empathy necessary to comprehend the emotional faces? The empathic effect on attentional mechanisms (eye movements), cortical correlates (N200 event-related potentials) and facial behaviour (electromyography) in face processing. Cogn Emot. 2016;30(2):210-224. doi:10.1080/02699931.2014.99 3306

35. Hofelich AJ, Preston SD. The meaning in empathy: distinguishing conceptual encoding from facial mimicry, trait empathy, and attention to emotion. Cogn Emot. 2012;26(1):119-128. doi:10.1080/026999 31.2011.559192

36. Cowan DG, Vanman EJ, Nielsen M. Motivated empathy: the mechanics of the empathic gaze. Cogn Emot. 2014;28(8): 1522-1530. doi:10.1080/02699931.2014.890563

37. Sun J, Liu P. The advantage of empathy from evidence of visual processing. Adv Psychol Sci. 2017;25(suppl):7.

38. Sun J, Liu P, Li D. Attentional characteristics of high-empathy people in processing emotional stimuli and evidence of eye movements. J Psychol Sci. 2018;41(5):1084-1089.

39. Choi D, Watanuki S. Effect of empathy trait on attention to faces: an event-related potential (ERP) study. J Physiol Anthropol. 2014;33 (1):4. doi:10.1186/1880-6805-33-4

40. MacLeod C, Mathews A, Tata P. Attentional bias in emotional disorders. J Abnorm Psychol. 1986;95(1):15-20. doi:10.1037/0021843X.95.1.15

41. MacLeod C, Mathews A. Anxiety and the allocation of attention to threat. $Q J$ Exp Psychol. 1988;40(4):653-670. doi:10.1080/ 14640748808402292

42. Koster EH, Crombez G, Verschuere B, De Houwer J. Selective attention to threat in the dot probe paradigm: differentiating vigilance and difficulty to disengage. Behav Res Ther. 2004;42(10):1183-1192. doi:10.1016/j.brat.2003.08.001
43. Zhang F, Dong Y, Wang K, Zhan Z, Xie L. Reliability and validity of the Chinese version of the interpersonal reactivity index-C. Chin $J$ Clin Psychol. 2010;18(2):155-157.

44. Chang H, Dong Y, Wang K, et al. The ability of self-face recognition and its relation to the empathy in patients with schizophrenia. Chin J Behav Med Brain Sci. 2012;21(3):193-195.

45. Zhang F, Dong Y, Wang K, et al. An association study of empathy and executive function in schizophrenic patients. Chin J Behav Med Brain Sci. 2010;19(5):408-411.

46. Yu W. Ability of empathy, personality and personal communication among medical graduates. Chin J Sch Health. 2013;34(1):40-42.

47. Cheng L, Yu X. Analysis on the reliability and validity of Chinese version of interpersonal reactivity index-C on nursing students during clinical practice. Mod Preven Med. 2014;41(15):2779-2780,2789.

48. Li W, Li C, Shen Y, Dan F. Effect of empathy on college students' altruistic behavior: a moderated mediation model. Psychol Devel Educ. 2015;31(5):571-577.

49. Song M, Zhou J, Ma J, et al. Relationship between empathy ability and perceived social support in military college students. Chin Ment Health J. 2019;33(2):149-152.

50. Meng J, Hu L, Shen L, et al. Emotional primes modulate the responses to others' pain: an ERP study. Exp Brain Res. 2012;220 (3-4):277-286. doi:10.1007/s00221-012-3136-2

51. Bai L, Ma H, Huang Y, Luo Y. The development of native Chinese affective picture system-a pretest in 46 college students. Chin Ment Health J. 2005;19(11):719-722.

52. Zhang G, Li A, Miao C, He X, Zhang M, Zhang Y. A consumer-grade LCD monitor for precise visual stimulation. Behav Res Methods. 2018;50(4):1496-1502. doi:10.3758/s13428018-1018-7

53. Gao X, Wang Q, Jackson T, Zhao G, Liang Y, Chen H. Biases in orienting and maintenance of attention among weight dissatisfied women: an Eye-Movement Study. Behav Res Ther. 2011;49 (4):252-259. doi:10.1016/j.brat.2011.01.009

54. Gao X, Wang Q-C, Chen H, Wang B-Y, Zhao G. Time course of attentional bias components toward body-shape related pictures among women with fat negative physical self: an Eye Movement Study. Acta Psychol Sin. 2012;44(4):498-510. doi:10.3724/SP. J.1041.2012.00498

55. Williams AC. Facial expression of pain: an evolutionary account. Behav Brain Sci. 2002;25(4):439-455.

56. $\mathrm{Gu} \mathrm{X}$, Han S. Attention and reality constraints on the neural processes of empathy for pain. NeuroImage. 2007;36(1):256-267. doi:10.1016/j.neuroimage.2007.02.025

57. Davis $\mathrm{MH}$. The effects of dispositional empathy on emotional reactions and helping: a multidimensional approach. J Pers. 1983;51 (2):167-184. doi:10.1111/j.1467-6494.1983.tb00860.x

58. Batson CD, Duncan BD, Ackerman P, Buckley T, Birch K. Is empathic emotion a source of altruistic motivation? J Pers Soc Psychol. 1981;40(2):290-302. doi:10.1037/0022-3514.40.2.290

59. Vuilleumier P. How brains beware: neural mechanisms of emotional attention. Trends Cogn Sci. 2005;9(12):585-594. doi:10.1016/j. tics.2005.10.011

60. Bi T, Du Y, Wang X, et al. Modulations of emotional attention and spatial attention on human visual cortical activities. Psychol Res Behav Manag. 2019;12:375-384. doi:10.2147/PRBM.S188121

61. Stanger N, Kavussanu M, Ring C. Put yourself in their boots: effects of empathy on emotion and aggression. J Sport Exerc Psychol. 2012;34(2):208-222. doi:10.1123/jsep.34.2.208

62. Pratto F, John OP. Automatic vigilance: the attention-grabbing power of negative social information. J Pers Soc Psychol. 1991;61 (3):380-391. doi:10.1037/0022-3514.61.3.380

63. Lang PJ, Bradley MM, Cuthbert BN. Motivated attention: affect, activation, and action. In: Attention and Orienting: Sensory and Motivational Processes. Mahwah, NJ, US: Lawrence Erlbaum Associates Publishers; 1997:97-135. 
64. Chikovani G, Babuadze L, Iashvili N, Gvalia T, Surguladze S. Empathy costs: negative emotional bias in high empathisers. Psychiatry Res. 2015;229(1-2):340-346. doi:10.1016/j.psychres.2015.07.001

65. Pool E, Brosch T, Delplanque S, Sander D. Attentional bias for positive emotional stimuli: a meta-analytic investigation. Psychol Bull. 2016;142(1):79-106. doi:10.1037/bu10000026

66. Batson CD. Empathy-induced altruistic motivation. In: Prosocial Motives, Emotions, and Behavior: The Better Angels of Our Nature. Washington, DC, US: American Psychological Association; 2010:15-34.

67. Morelli SA, Lieberman MD, Zaki J. The emerging study of positive empathy. Soc Personal Psychol Compass. 2015;9(2):57-68. doi:10. $1111 / \mathrm{spc} 3.12157$

68. Calvo MG, Lang PJ. Gaze patterns when looking at emotional pictures: motivationally biased attention. Motiv Emot. 2004;28 (3):221-243. doi:10.1023/B:MOEM.0000040153.26156.ed

69. Nummenmaa L, Hyönä J, Calvo MG. Eye movement assessment of selective attentional capture by emotional pictures. Emotion. 2006;6 (2):257-268. doi:10.1037/1528-3542.6.2.257

70. Hajcak G, Olvet DM. The persistence of attention to emotion: brain potentials during and after picture presentation. Emotion. 2008;8 (2):250-255. doi:10.1037/1528-3542.8.2.250

71. Fan Y, Han S. Temporal dynamic of neural mechanisms involved in empathy for pain: an Event-Related Brain Potential Study. Neuropsychologia. 2008;46(1):160-173. doi:10.1016/j. neuropsychologia.2007.07.023

72. Han S, Fan Y, Mao L. Gender difference in empathy for pain: an electrophysiological investigation. Brain Res. 2008;1196:85-93. doi:10.1016/j.brainres.2007.12.062

73. Pessoa L, Kastner S, Ungerleider LG. Attentional control of the processing of neutral and emotional stimuli. Cogn Brain Res. 2002;15(1):31-45. doi:10.1016/S0926-6410(02)00214-8

74. Vuilleumier P, Armony JL, Driver J, Dolan RJ. Effects of attention and emotion on face processing in the human brain: an event-related fMRI Study. Neuron. 2001;30(3):829-841. doi:10.1016/S08966273(01)00328-2

75. van Zonneveld L, Platje E, de Sonneville L, van Goozen S, Swaab H. Affective empathy, cognitive empathy and social attention in children at high risk of criminal behaviour. $J$ Child Psychol Psychiatry. 2017;58(8):913-921. doi:10.1111/jcpp.12724
76. Schmukle SC. Unreliability of the dot probe task. Eur J Pers. 2005;19(7):595-605. doi:10.1002/per.554

77. Chapman A, Devue C, Grimshaw GM. Fleeting reliability in the dot-probe task. Psychol Res. 2019;83(2):308-320. doi:10.1007/ s00426-017-0947-6

78. Staugaard SR. Reliability of two versions of the dot-probe task using photographic faces. Psychol Sci. 2009;51(3):339-350.

79. Aday JS, Carlson JM. Extended testing with the dot-probe task increases test-retest reliability and validity. Cogn Process. 2019;20 (1):65-72. doi:10.1007/s10339-018-0886-1

80. Price RB, Kuckertz JM, Siegle GJ, et al. Empirical recommendations for improving the stability of the dot-probe task in clinical research. Psychol Assess. 2015;27(2):365-376. doi:10.1037/pas 0000036

81. Zhou Q, Valiente C, Eisenberg N. Empathy and its measurement. In: Lopez SJ, Snyder CR, editors. Positive Psychological Assessment: A Handbook of Models and Measures. American Psychological Association; 2003:269-284.

82. Hastings PD, Miller JG, Kahle S, Zahn-Waxler C. The neurobiological bases of empathic concern for others. In: Killen M, Smetana JG, editors. Handbook of Moral Development. Psychology Press; 2014:411-434.

83. Hein G, Lamm C, Brodbeck C, Singer T. Skin conductance response to the pain of others predicts later costly helping. PLoS One. 2011;6 (8):e22759. doi:10.1371/journal.pone.0022759

84. Rae Westbury H, Neumann DL. Empathy-related responses to moving film stimuli depicting human and non-human animal targets in negative circumstances. Biol Psychol. 2008;78(1):66-74. doi:10. 1016/j.biopsycho.2007.12.009

85. Bons D, van den Broek E, Scheepers F, Herpers P, Rommelse N, Buitelaaar JK. Motor, emotional, and cognitive empathy in children and adolescents with autism spectrum disorder and conduct disorder. J Abnorm Child Psychol. 2013;41(3):425-443. doi:10.1007/s10802012-9689-5

86. Oliver LD, Mitchell DGV, Dziobek I, et al. Parsing cognitive and emotional empathy deficits for negative and positive stimuli in frontotemporal dementia. Neuropsychologia. 2015;67:14-26. doi:10.10 16/j.neuropsychologia.2014.11.022
Psychology Research and Behavior Management

\section{Publish your work in this journal}

Psychology Research and Behavior Management is an international, peer-reviewed, open access journal focusing on the science of psychology and its application in behavior management to develop improved outcomes in the clinical, educational, sports and business arenas. Specific topics covered in the journal include: Neuroscience, memory and decision making; Behavior modification and management; Clinical

\section{Dovepress}

applications; Business and sports performance management; Social and developmental studies; Animal studies. The manuscript management system is completely online and includes a very quick and fair peer-review system, which is all easy to use. Visit http://www. dovepress.com/testimonials.php to read real quotes from published authors. 